\title{
A Systematic Literature Review of Factors Affecting Cloud Computing Adoption Internationally
}

\author{
Raja Muhammad Ubaid Ullah ${ }^{\# 1}$, Dr.Kevan A. Buckley ${ }^{\# 2}$,Dr. Mary Garvey ${ }^{\# 3}$, Dr. Jun Li ${ }^{\# 4}$. \\ School of Mathematics and Computer Science \\ University of Wolverhampton, Wulfruna Street, Wolverhampton WVI ILY, UK.
}

\begin{abstract}
Context: The adoption of cloud computing in small and medium size organisations has become a significant trend in today's information technology. Therefore, there is a requirement to identify possible security challengesand guidelines for the researchers based on the adoption. Keeping in view of these elements, a systematic literature review (SLR) of cloud computing adoption in businesses and organisations was conducted.
\end{abstract}

Goal: The main purpose of this research is to identify the possible opportunities, challenges, factors and guidelines for the evaluation and adoption of cloud computing (CC) for organisations intending to adopt or already using $C C$.

Method: ASLR is conducted to achieve the above goals.The search process of this literature review used quality scholarly databases such as ACM Digital Library, IEEE Xplore, Inspec, Springer, Google Scholar, Researchgate, Google Books and Wolverhampton University Online Library(8 types of databases) along with open-access journals for relevant articles.

Results: A total of 561articles were found of which 85 articles met the included criteria. Ninedifferent categorieswere identified securingCCadoption in organisation, namely (1) CC adoption ( $n=30)$; (2) CC security ( $n=18) ;$ (3) CC overview / Objective $(n=8) ;(4)$ CC adoption challenges $(n=7) ;(5) C C$ adoption factor influencing ( $n=7) ;(6) C C$ adoption factor affecting ( $n=6)$; (7) CC future forecast $(n=4)$; (8) CC adoption decision ( $n=3)$; (9) CC adoption \& implementation $(n=2)$;

Conclusion:The basis of this SLRcan help the development of guidelines to support newcomer companies whether to adopt and migrate to $C C$, the evaluation of relationship between nine factors and the selection of providers.

Keywords - cloud computing security, cloud computing adoption

\section{INTRODUCTION}

A SLR of cloud based enterprise solutions was conducted with respect to their adoption. The primary goal was to identify the factors and challenges from existing literature, which influence the adoption process of CC services in organisations and areas that have been researched with respect to the adoption processes [16]. In enterprises the adoption of CC is considered as cost effective and secure use of information and communication technologies. The objectives of organisations are to improve the cooperation and co-ordination, the quality of business services, and to reduce the cost of different types of IT services at the same time, by using $\mathrm{CC}$ more effectively and securely [3, p.34-37, 90].

Literature reviewing is an imperative process of research, which facilitates the identification of areas and their respective issues in which more research is needed [163]. So the review process has been followed using the fundamental guidelines for conducting a successful literature review within certain boundaries $[163,164,169]$. However there are considerable issues that really need to be addressed before they can use the services of CC [166].

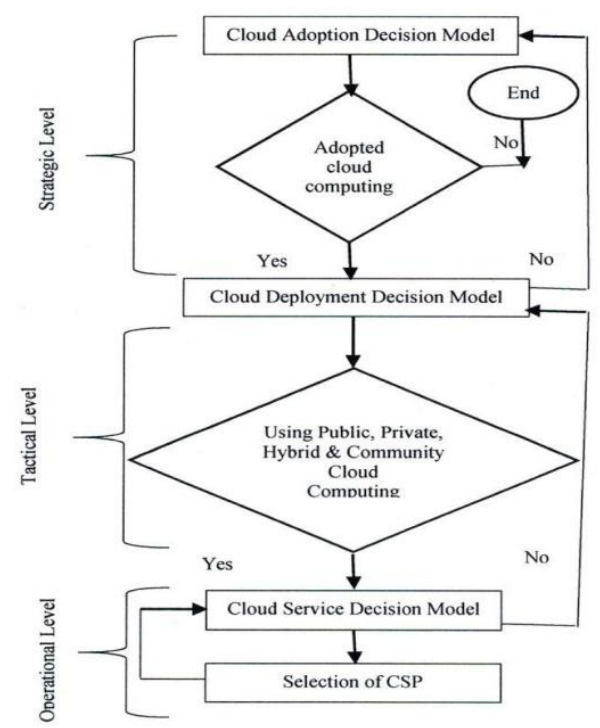

Figure 1: Flow Chart of Cloud Adoption Decision Model. 
The main features that differentiate a SLR from a traditional literature review are:

- SLR addresses the specified research questions by defining a review protocol.

- Systematic review defines the search strategy that aims to detect as much of the relevant literature as possible.

- Systematic reviews require inclusion and exclusion criteria to assess each potential primary study.

The guidelines and systematic process in this research workwere adopted [113]. The systematic review was conducted mainly in three phases:

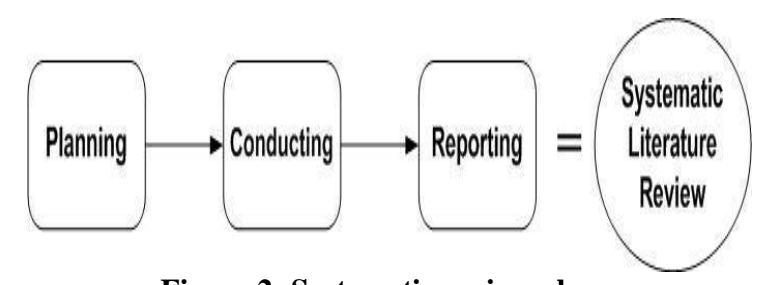

Figure 2: Systematic review phases.

- Planning the review: Associated with identification of the need for a review and developing the review protocol.

- Conducting the review: Associated with selection of primary studies, quality assessment, data extraction and data synthesis.

- Reporting the review: Associated with reporting the results and documenting the process.

The appropriate use of Information and Communication Technologies (ICT) helps businesses and organisation to become more efficient [40]. CC facilitates the organisations by delivering resources and respective services by a user-pay system through the Internet [176]. CCmainly offers a new business solution, which enables the organisations to rent the required information technology (IT) infrastructure, which includes respective platforms, the software through positioning their business applications and their data storage in the cloud environment. There are many well-known cloud examples which include Amazon, Google and Microsoft (Department of Finance and Deregulation (DFD), [174].

Many challenges are faced by the organisations and businesses according to their size and structure. In comparison to large firms, small firms have less cost tolerance and risk of adopting new innovations. In any organisationIT investment comprises different types of costs such as fixed costs, operation costs $\&$ training costs. In some cases the actual cost of a project goes higher than the initial estimates [41].
Unsurprisingly, opportunities and savings have attracted strong market growth in such services. [175] The worldwide public cloud services market is projected to grow 17.3 percent in 2019 to total $\$ 206.2$ billion, up from $\$ 175.8$ billion in 2018 , according to Gartner, Inc. In 2018, Gartner forecasts that the market will grow 21 percent, up from $\$ 145.3$ billion in 2017. The fastest-growing segment of the market is cloud system infrastructure services (infrastructure as a service or IaaS), which is forecast to grow 27.6 percent in 2019 to reach $\$ 39.5$ billion, up from $\$ 31$ billion in 2018 (see Table 1).

Table 1. Gartner Forecasts Worldwide Public Cloud Revenue to Grow 17.3 Percent in 2019.

\begin{tabular}{|l|l|l|l|l|l|}
\hline & $\mathbf{2 0 1 7}$ & $\mathbf{2 0 1 8}$ & $\mathbf{2 0 1 9}$ & $\mathbf{2 0 2 0}$ & $\mathbf{2 0 2 1}$ \\
\hline $\begin{array}{l}\text { Cloud Business Process Services } \\
\text { (BPaaS) }\end{array}$ & 42.2 & 46.6 & 50.3 & 54.1 & 58.1 \\
\hline $\begin{array}{l}\text { Cloud Application Infrastructure } \\
\text { Services (PaaS) }\end{array}$ & 11.9 & 15.2 & 18.8 & 23.0 & 27.7 \\
\hline Cloud Application Services (SaaS) & 58.8 & 72.2 & 85.1 & 98.9 & 113.1 \\
\hline $\begin{array}{l}\text { Cloud Management and Security } \\
\text { Services }\end{array}$ & 8.7 & 10.7 & 12.5 & 14.4 & 16.3 \\
\hline $\begin{array}{l}\text { Cloud System Infrastructure } \\
\text { Services (IaaS) }\end{array}$ & 23.6 & 31.0 & 39.5 & 49.9 & 63.0 \\
\hline Total Market & $\mathbf{1 4 5 . 3}$ & $\mathbf{1 7 5 . 8}$ & $\mathbf{2 0 6 . 2}$ & $\mathbf{2 4 0 . 3}$ & $\mathbf{2 7 8 . 3}$ \\
\hline
\end{tabular}

Note: Totals may not add up due to rounding.

\section{GUIDELINES FOR SYSTEMATIC LITERATURE REVIEW}

The guidelines for performing SLR in this particular related subject area [113] were followed for technology related issues. After include/exclude criteria and quality assessment criteria were identified, a chosen set of articles were available for the data extraction process. The reviewed articles were categorised according to the research methods used in them to specifically determine the extent of CC adoption research [170, 171, 165]. Particularly focusing on the research methods applied in the reviewed articles included journal, articles, case studies, surveys, research papers, conceptual papers, press releases, workshops, $\mathrm{PhD}$ theses, conference publications and books chapter (11 types of articles) in related areas. Some related articles did not have any sort of methodology section, but they reflect on some concepts in relation to CC (e.g., cost, security, performance, etc.) or they adopt theories without empirical testing. These types of papers are labelled as a conceptual papers.

Classification of the 85 selected articles in manufacturing, health, education, warehousing, engineering, telecommunication, construction and research sectors involved using a thorough and theoretical analysis of the relevant topic as suggested by $[172,173]$. Therefore, the process of classification resulted in 11 corresponding variables (i.e., external 
support, competitive pressure, seniormanagement support, employees cloud knowledge, adequate resources, information intensity, relative advantage, complexity, compatibility, security / privacy and cost effectiveness). Finally, selective coding was applied to integrate and refine the 4 main categories (environmental, human, organisational and technological) and their 11 respective variables to develop relationships between them $[172,173]$.

Without clearly defined regulations, some countries also restrictedtheir enterprises to store their particular valuable data in a CC infrastructure within their respective national borders [11]. For instance, the EU's privacy laws clearly prohibit the give-andtake of personal information outside the user's jurisdiction [13]. As the Cloud Service Provider's (CSP) which operate outside of their home country must act in accordance with the host country's regulations and government surveillance, a ruling that some of enterprises may find very difficult to follow [14, 114, 144]. Multi-jurisdictional politics is an example that can have adverse impact on the adoption of CC in the USA Patriot Act, which have a doubt for dealing with US-based CSP's for the countries, especially those within the EU $[115,162]$. However some CSP's have solved this particular issue by permitting enterprises to set up their IT resources on physical servers, which are located within the EU region [115].

Keystones towards the adoption of CC is the IT knowledge, competence, and capability that seniormanagement needs to contribute for creating a suitable organisational environment. Such environments must offer sufficient budgets, adequate human and IT resources, and more time [116, 117 , 118, 119]. This involves:

1) Understanding $\mathrm{CC}$ and its architecture, service models, \& strategic values $[120,121,122,123$, 124, 125];

2) Identifying an enterprise's business needs \& aligning IT decisions with business strategies $[13,126]$;

3) Evaluating the readiness of the existing IT infrastructure, IT knowledge, and human resource skills, available resources, and culture [124, 121, 117, 122]; and

4) Steering towards CC adoption (e.g. deciding on an adoption strategy, governance of integration, implementation, and evaluation of $\mathrm{CC}$ services after use) with the guidance of external regulatory \& professional bodies [127, 13].

The significant influence of the perceived factors and their related variables to the adoption of CC services $[118,128]$. This perspective is especially with the finding of literature review that found the management's perceptions towards security and privacy, cost evaluation, and adequate resources to have a substantial impact on the decision to adopt CC [129]. These benefits comprise cost savings, agility, flexibility, ease of use, scalability, the ease of cooperation between business partners, based on less operational effort on the part of CIO's, and increased productivity [117, 130,116, 132, 131, 126, 133]. However, by proofing the concept of CC services, the enterprises are capable to identify risks and benefits so they can decide whether or not to adopt CC. The identified risks in this review include [134, 11, 132, 131, 135, 128, 115]:

- Organizational risks, which cover the risk of vendor lock-in as well as the loss of governance within the enterprise.

- Technical risks, which include data leakage, loss of data, downtime, data bottlenecks, and cyber-attacks.

- Legal risks, which include data protection regulations and licensing issues.

- Nontechnical risks, which refer to the misuse of cloud services and natural disasters.

- Performance risks, which primarily result from the moving of huge amounts of data to cloud servers. This movement can take a long time and when moving further in the adoption, it may require increasing bandwidth and connectivity, which is costly [127].

- Security risks, some of these risks are linked to weakcloud securitymeasures of the services. Storing data without encryption and lack of multi-factor authentication for access can lead to loss of intellectual property, loss of management control, exposure to malware, compliance violations, contractual breaches with customers and partners and ultimately loss of customer trust and loss of revenue [200].

\section{METHODS}

A SLRrequires a comprehensive and fair analysis of particular searched literature. It maximises the extent of particular searched literature that identifies some of the most possible used alternative words or concepts and synonyms in the particular research. [165]. Firstly, the manual search is conducted in the related areas such as Computer science and businesses. The search process of this literature review used the 8 quality scholarly selected types of databases. These databases facilitated to cover more broad scope, openaccess which lead to Information System (IS) and11 types of articles in the relevant areas [164]. Whereas, online databases are the proper and practical sources for the reviewing of literature about modern day phenomenon such as CC [167].

The year of publication cannot be restricted, since cloud computing adoption was proposed only in the 
last ten years (i.e. 2009 to September 2018). The search criteria was limited to the 11 types of articles in order to ensure their relevance. After the general study of related areas, the language was limited to English. Furthermore, the search strings terms that are used for searching all the 8types of databases were $\mathrm{CC}$ in combination with adopt* and other respective related terms, such as accept*, cost*, diffuse*, security*, secure*, evaluation*, and guidelines*.

The initial search string in all the mentioned databases resulted in 561articles. In order to focus on the most relevant literature, the process of practical screening was conducted.Primary evaluation involved the reading of abstract and their table of contents of selected articles to decide whether or not they are relevant to this review $[169,168]$. Hence, the evaluation is based on the criteria specified in Table 2. On the other hand, the criteria of filtering involved the exclusion of recurring papers, research-inprogress papersand non-English language papers. These particularly focussed on individuals and periodical articles published by news websites, trade journals, and magazines. The independent criteria of inclusion was applied for each particular author to select the relevant articles and books. This helpedto delimit the sample of articlesso that the literature review is practically manageable [168]. Through this process of evaluation 85articleswere selected for thorough study and classification of articles was included in the reference list.

\begin{tabular}{|l|c|}
\hline Include criteria & Exclude criteria \\
\hline • Directly or indirectly & - Irrelevant to study of \\
related to both businesses & the cloud or businesses. \\
and cloud technology. & - Conceptual methods or \\
- Cloud-based businesses & cognitive introductions. \\
frameworks design. & - Review papers. \\
- Cloud computing solutions & - Business analysis \\
$\begin{array}{l}\text { applied in businesses. } \\
\text { Security and privacy } \\
\text { mechanisms of businesses } \\
\text { data in cloud. }\end{array}$ & reports. \\
- Written in English. & \\
\hline
\end{tabular}

Table 2:Selected articles related to the criteria based on search Databases.

\begin{tabular}{|l|c|c|c|c|}
\hline \multicolumn{1}{|c|}{ Database } & $\begin{array}{c}\text { Total } \\
\text { No. of } \\
\text { Research }\end{array}$ & $\begin{array}{c}\text { No. of } \\
\text { Excluded } \\
\text { Studies }\end{array}$ & $\begin{array}{c}\text { No. of } \\
\text { Selected } \\
\text { Studies }\end{array}$ & $\begin{array}{c}\text { Per \% } \\
\text { of } \\
\text { Selected } \\
\text { Studies }\end{array}$ \\
\hline $\begin{array}{l}\text { ACM Digital } \\
\text { Library }\end{array}$ & 64 & 52 & 12 & $18.75 \%$ \\
\hline IEEE Xplore & 83 & 70 & 13 & $15.66 \%$ \\
\hline Inspec & 45 & 34 & 11 & $24.44 \%$ \\
\hline Springer Link & 101 & 87 & 14 & $13.86 \%$ \\
\hline Google Scholar & 81 & 71 & 10 & $12.35 \%$ \\
\hline Google Books & 35 & 33 & 2 & $5.71 \%$ \\
\hline Researchgate & 65 & 51 & 14 & $21.54 \%$ \\
\hline $\begin{array}{l}\text { Wolverhampton } \\
\text { University } \\
\text { Online Library }\end{array}$ & 87 & 78 & 9 & $10.34 \%$ \\
\hline \multicolumn{1}{|c|}{ Total --> } & 561 & 476 & 85 & $15.15 \%$ \\
\hline
\end{tabular}

\section{$\underline{\text { Result: } \text { A Statistical Analysis }}$}

\section{CONTINENT WISE PUBLICATIONS}

The geographical classification of the publications across five Continents highlights that the Asian Continent has the highest number in research with $40 \%$ of the articles. In second position Europe Continent has $30.59 \%$ of the articles. In third position American Continent has $20 \%$ of the articles. At fourth position African Continent has $5.88 \%$ of the articles and finally the Oceania Continent has $3.53 \%$ of the articles. Table 3 reflects the picture of the research articles published Continent-wise, which shows that Asia, Europe and America have a significant share in research, whereas Africa and Oceania both have a proportionally lower portion of share in the research.

Table 3: Continent wise publications.

\begin{tabular}{|l|c|r|}
\hline Continents & No. of Articles & \multicolumn{1}{|c|}{ Per \% } \\
\hline Asia & 34 & $40.00 \%$ \\
\hline Europe & 26 & $30.59 \%$ \\
\hline America & 17 & $20.00 \%$ \\
\hline Africa & 5 & $5.88 \%$ \\
\hline Oceania & 3 & $3.53 \%$ \\
\hline & & \\
\hline Total --> & 85 & $100.00 \%$ \\
\hline
\end{tabular}

As shown in the Table 4 Asiahas overall $40 \%$ contribution of the total articles. Within Asia,India is top on the list with $38.24 \%$, followed by Malaysia with $35.29 \%$ and Taiwan in third position with $5.88 \%$. Whereas, Bangladesh, China, Jordan, Pakistan, Saudi Arabia, Singapore and Vietnam with $2.94 \%$ each one published articles respectively.

\section{A. Asian Continent}

Table 4: Asian Continent publications count.

\begin{tabular}{|l|c|c|}
\hline Countries & No of Articles & Per \% \\
\hline India & 13 & $38.24 \%$ \\
\hline Malaysia & 12 & $35.29 \%$ \\
\hline Taiwan & 2 & $5.88 \%$ \\
\hline Bangladesh & 1 & $2.94 \%$ \\
\hline China & 1 & $2.94 \%$ \\
\hline Jordan & 1 & $2.94 \%$ \\
\hline Pakistan & 1 & $2.94 \%$ \\
\hline Saudi Arabia & 1 & $2.94 \%$ \\
\hline Singapore & 1 & $2.94 \%$ \\
\hline Vietnam & 1 & $2.94 \%$ \\
\hline \multicolumn{3}{|l}{} \\
\hline Total --> & 34 & $100.00 \%$ \\
\hline
\end{tabular}

Europe has $30.59 \%$ contribution with second on the table of articles publication internationally. As shown in Table 5UK is on top with $30.77 \%$, surprisingly Ireland is on second position with $11.54 \%$. France, 
Portugal, Austria and Germany with $7.69 \%$ each one on third position in article publications showing their interest in Cloud Computing. Whereas, Finland, Italy, Lativa, Norway, Romania, Scotland and Slovenia play a secondary role by contributing with $3.85 \%$ each one in the publication for our analysis.

\section{B. Europe Continent}

Table 5: Europe Continent publications count.

\begin{tabular}{|l|c|c|}
\hline Countries & No of Articles & Per \% \\
\hline UK & 8 & $30.77 \%$ \\
\hline Ireland & 3 & $11.54 \%$ \\
\hline Austria & 2 & $7.69 \%$ \\
\hline France & 2 & $7.69 \%$ \\
\hline Germany & 2 & $7.69 \%$ \\
\hline Portugal & 2 & $7.69 \%$ \\
\hline Finland & 1 & $3.85 \%$ \\
\hline Italy & 1 & $3.85 \%$ \\
\hline Lativa & 1 & $3.85 \%$ \\
\hline Norway & 1 & $3.85 \%$ \\
\hline Romania & 1 & $3.85 \%$ \\
\hline Scotland & 1 & $3.85 \%$ \\
\hline Slovenia & 1 & $3.85 \%$ \\
\hline \multicolumn{3}{|l}{} \\
\hline Total --> & 26 & $100.00 \%$ \\
\hline
\end{tabular}

Table 6 shows that American Continent has a 20\% contribution internationally. The U.S. dominates in the research with $94.12 \%$ of the articles and a very marginal role of Canada with $5.88 \%$.

\section{American Continent}

Table 6: American Continent publications count.

\begin{tabular}{|l|c|c|}
\hline Countries & No of Articles & Per \% \\
\hline USA & 16 & $94.12 \%$ \\
\hline Canada & 1 & $5.88 \%$ \\
\hline \multicolumn{3}{|c|}{} \\
\hline Total --> & 17 & $100.00 \%$ \\
\hline
\end{tabular}

Table 7 represents that African Continent has a 5.88\% share on the publication of articles internationally. The top on the list is Kenya with $40 \%$ followed by Ghana, Nigeria and South Africa playing a secondary role by contributing with $20.00 \%$ each one with the publication.

\section{African Continent}

Table 7: African Continent publications count.

\begin{tabular}{|l|c|c|}
\hline Countries & No of Articles & Per \% \\
\hline Kenya & 2 & $40.00 \%$ \\
\hline Ghana & 1 & $20.00 \%$ \\
\hline Nigeria & 1 & $20.00 \%$ \\
\hline South Africa & 1 & $20.00 \%$ \\
\hline \multicolumn{3}{|l}{} \\
\hline Total --> & 5 & $100.00 \%$ \\
\hline
\end{tabular}

Table 8 shows that Oceania Continent is on the bottom of the table with $3.53 \%$ contribution of publication internationally. Australia is the only Country which contributed in this continent that published 3 articles.

\section{E. Oceania Continent}

Table 8: Oceania Continent publications count.

\begin{tabular}{|l|c|c|}
\hline Country & No of Articles & Per \% \\
\hline Australia & 3 & $100.00 \%$ \\
\hline \multicolumn{3}{|c|}{} \\
\hline Total --> & 3 & $100.00 \%$ \\
\hline
\end{tabular}

\section{PUBLICATION YEAR DISTRIBUTION}

Table 9 shows data on yearly basis of the selected primary articles. As we have time restriction applied on the study, we found no significant articles published before 2009. The Cloud Computing term was coined in year 2006 and at the end of 2008 mostly researchers started to widely consider this important topic. Therefore, we do limit the year of articles considered in our research range from 2009 to first three quarters of September 2018.

The study cover the ten years of the period, interestingly the up and down flow of the data is encouraging, which reflects that this research area is still going on positively. Closely focusing at the publication year distribution, out of all the selected articles, we found only 2 articles $(2.35 \%)$ which were published in 2009, 3 articles $(3.53 \%$ ) in 2010, more interest of the research started from 2011 when 7 articles $(8.24 \%)$ were published, then 11 articles (12.94\%) were published simultaneously in 2012, 2013 and 2014. In 2015 publications dropped to 7 articles (8.24\%), but from 2016 trend of publication started going up with 9 articles (10.59\%), 10 articles (11.76\%) were published in 2017 and finally 14 articles $(16.47 \%)$ were published in the first three quarters (i.e. up to month of September) of 2018.

Surprisingly this shows good increasing in number of the publications each year, which clearly suggests a quiet good number of growing interest in CC adoption. It also shows that trust is developing on $\mathrm{CC}$ environment and more companies are confidently using or intend to use this technology in near future.

\section{Year Wise No. Of Articles Selected}

Table 9:Number of articles across the years.

\begin{tabular}{|c|c|r|}
\hline Years & No. of Articles & Per \% \\
\hline 2018 & 14 & $16.47 \%$ \\
\hline 2017 & 10 & $11.76 \%$ \\
\hline 2016 & 9 & $10.59 \%$ \\
\hline 2015 & 7 & $8.24 \%$ \\
\hline 2014 & 11 & $12.94 \%$ \\
\hline
\end{tabular}




\begin{tabular}{|c|c|r|}
\hline 2013 & 11 & $12.94 \%$ \\
\hline 2012 & 11 & $12.94 \%$ \\
\hline 2011 & 7 & $8.24 \%$ \\
\hline 2010 & 3 & $3.53 \%$ \\
\hline 2009 & 2 & $2.35 \%$ \\
\hline \multicolumn{3}{|c|}{} \\
\hline Total --> & 85 & $100.00 \%$ \\
\hline
\end{tabular}

\section{PUBLICATION CHANNEL}

Table 10 shows the selected articles of different types of publications. In this study publication type means the channel where the selected articles has been published. Variety of publication types included in this study for instance, conferences, journal, articles, conceptual papers, research papers, surveys, case study, press release, $\mathrm{PhD}$ theses, publication, workshop and book chapter also to cover every aspect of the study regarding the $\mathrm{CC}$ adoption. Interestingly majority of the articles were published in conferences $33(38.82 \%)$, the journals are second 23 (27.06\%) publications, third position is hold by articles $15 \quad(17.65 \%)$ publications. Whereas, conceptual and research papers were $3(3.53 \%)$ each one published accordingly. The rest of the selected articles published are not encouraging in numbers, such as surveys $2(2.35 \%)$ publications and case study, press release, $\mathrm{PhD}$ theses, publication, book chapter and workshop selected $1(1.18 \%)$ each published accordingly.

Table 10: Selected articles with publication types.

\begin{tabular}{|c|c|c|c|}
\hline $\begin{array}{l}\text { Publication } \\
\text { Type }\end{array}$ & $\begin{array}{l}\text { Publication Type } \\
\text { Description }\end{array}$ & $\begin{array}{c}\text { No of } \\
\text { Articles }\end{array}$ & Per \% \\
\hline $\mathrm{CON}$ & Conferences & 33 & $38.82 \%$ \\
\hline JOR & Journals & 23 & $27.06 \%$ \\
\hline ART & Articles & 15 & $17.65 \%$ \\
\hline $\mathrm{CP}$ & Conceptual Papers & 3 & $3.53 \%$ \\
\hline $\mathrm{RP}$ & Research Papers & 3 & $3.53 \%$ \\
\hline $\begin{array}{l}\text { SUR } \\
\end{array}$ & Surveys & 2 & $2.35 \%$ \\
\hline$\overline{B K}$ & Books & 1 & $1.18 \%$ \\
\hline $\mathrm{CS}$ & Case Study & 1 & $1.18 \%$ \\
\hline PRL & Press Release & 1 & $1.18 \%$ \\
\hline PTH & PhD Theses & 1 & $1.18 \%$ \\
\hline PUB & Publications & 1 & $1.18 \%$ \\
\hline WRK & Workshop & 1 & $1.18 \%$ \\
\hline \multicolumn{2}{|c|}{ Total articles selected --> } & 85 & $100.00 \%$ \\
\hline
\end{tabular}

\section{CLASSIFICATION OF THE RELEVANT ARTICLES}

Table 11 presents the classification of the relevant selected primary articles for this study. After carefully reading all the relevant selected articles and creating the different classifications based on the facts findings in accordance with the $\mathrm{CC}$ adoption. We identified that the majority of the relevant selected articles were related to the CC adoption (such as 30 with $35.39 \%$ ). This shows that still more and more businesses are interested in adoption of CC instead of traditional IT. In addition study also identified that the importance of the CC security (such as 18 with $21.18 \%$ ) is still in businesses priority, therefore security is an essential aspect of the CC for the safer environment. A substantial part of the articles on security highlighting a series of some new adoption problems. The third factor considered by the businesses is the CC overview / objectives (such as 8 with 9.41\%), which reflects that they are keen to know the pros and cons before adoption. Surprisingly the CC challenges and factor influencing (such as 7 with $8.24 \%$ ) each are both at same priority while considering the CC adoption respectively for the safer utilisation of platform. This showing that researchers still have interest in the challenges and factor influencing in the $\mathrm{CC}$ adoption consideration. Another important part of the study is the $\mathrm{CC}$ adoption factor affecting also been considered (such as 6 with $7.06 \%$ ) is not ignorable in the consideration of $\mathrm{CC}$ adoption for the smooth running of business environment and still need attention for researcher. The $\mathrm{CC}$ future forecast is also another factor considered by the researcher (such as 4 with $4.71 \%$ ) to keep eyes on the trend of this new technology. The CC adoption decision is been considered marginally low (i.e. 3 with $3.53 \%$ ) as research on this side not properly focused on this factor. Finally CC adoption \& implementation is still need attention for the researcher to address this issue as (2 with $2.35 \%)$ work is done on this area.

Table 11:Selected studies relevant to the articles.

\begin{tabular}{|l|c|c|}
\hline $\begin{array}{l}\text { Selected relevant } \\
\text { articles for articles. }\end{array}$ & $\begin{array}{l}\text { No. of } \\
\text { Articles }\end{array}$ & Per \% \\
\hline CC Adoption. & 30 & $35.29 \%$ \\
\hline CC Security. & 18 & $21.18 \%$ \\
\hline $\begin{array}{l}\text { CC Overview / } \\
\text { Objective. }\end{array}$ & 8 & $9.41 \%$ \\
\hline $\begin{array}{l}\text { CC Adoption } \\
\text { Challenges. }\end{array}$ & 7 & $8.24 \%$ \\
\hline CCA Factor Influencing. & 7 & $8.24 \%$ \\
\hline CCA Factor Affecting. & 6 & $7.06 \%$ \\
\hline CC Future Forecast. & 4 & $4.71 \%$ \\
\hline CC Adoption Decision. & 3 & $3.53 \%$ \\
\hline CCA \& Implementation. & 2 & $2.35 \%$ \\
\hline \multicolumn{2}{|l|}{} \\
\hline Total --> & 85 & $100.00 \%$ \\
\hline
\end{tabular}

$* \mathrm{CC}=$ Cloud Computing.

\section{RESULTS}

The results of our SLR reflect in four ways such as Continent wise, Countries wise, Year wise and details of their different mode of articles. After the searching steps, the evaluation and alternate reviews, finally selected 85 articles from the total reviewed of 561 articles, which were found in the first search. The selected 85 articles mostly cover the basic primary view of the studied area of CC adoption with organisation requirements. Hence, the organisation and $\mathrm{CC}$ areas have potential in future, therefore study 
results can offer the researchers up-to-date aspects for their research.

The above discussed empirical findings based on the four factors (such as, environmental, human, organisational and technological) and their respective 11 variables are outcome from our SLR. Methodological and theoretical findings indicate that few number of qualitative studies have contributed toward the understanding of $\mathrm{CC}$ adoption factors as compared with quantitative studies. Whereas, in some articles, several methods are used [142, 137, 117, 138]. Furthermore, factors and their respective variables regarding the adoption of CC are extensively addressed in certain articles. In general, processes of cloud adoption, such as external support, competitive pressure, senior management support, employees cloud knowledge, adequate resources, information intensity, relative advantage, complexity, compatibility, security / privacy and cost effectiveness are not adequately addressed, except for the proof of concept process.

In the study phase we found 30 countries where different types of research articles were published on CC enterprise (See Table 12 review articles classifications and Table 13 based on the type and references). The criteria based on all the selected searched articles, which are published from 2009, and this may specify that the research in CC for different types of businesses is still an emerging area. The number of articles which are published across years in our searched results are shown in Table 9.

Table 12. Countries conducting cloud-based business researches.

\begin{tabular}{|c|c|c|}
\hline Country & $\begin{array}{l}\text { No. of } \\
\text { articles }\end{array}$ & References \\
\hline USA & 16 & $\begin{array}{l}10,15,25,81,95,114,126,129, \\
130,135,136,156,158,175, \\
191,196\end{array}$ \\
\hline India & 13 & $\begin{array}{l}2,6,14,18,89,109,116,123 \\
124,125,128,197,198\end{array}$ \\
\hline Malaysia & 12 & $\begin{array}{l}133,144,151,154,155,179 \\
180,185,188,189,194,199\end{array}$ \\
\hline UK & 8 & $\begin{array}{l}4,118,119,120,152,186,187, \\
195\end{array}$ \\
\hline Australia & 3 & $157,176,193$ \\
\hline Ireland & 3 & $13,127,145$ \\
\hline France & 2 & 117,142 \\
\hline Portugal & 2 & 122,150 \\
\hline Taiwan & 2 & 131,140 \\
\hline Austria & 2 & 66,181 \\
\hline Germany & 2 & 9,11 \\
\hline Kenya & 2 & 26,183 \\
\hline Bangladesh & 1 & 7 \\
\hline Canada & 1 & 143 \\
\hline China & 1 & 92 \\
\hline Finland & 1 & 121 \\
\hline Ghana & 1 & 146 \\
\hline Italy & 1 & 192 \\
\hline Jordan & 1 & 177 \\
\hline Latvia & 1 & 182 \\
\hline
\end{tabular}

\begin{tabular}{|l|l|l|}
\hline Nigeria & 1 & 134 \\
\hline Norway & 1 & 16 \\
\hline Pakistan & 1 & 8 \\
\hline Romania & 1 & 115 \\
\hline Saudi Arabia & 1 & 184 \\
\hline Scotland & 1 & 82 \\
\hline Singapore & 1 & 28 \\
\hline Slovenia & 1 & 190 \\
\hline South Africa & 1 & 138 \\
\hline Vietnam & 1 & 132 \\
\hline
\end{tabular}

Table 13. Types of Articles with References.

\begin{tabular}{|l|c|l|}
\hline $\begin{array}{c}\text { Types of } \\
\text { Article }\end{array}$ & $\begin{array}{c}\text { No of } \\
\text { Articles }\end{array}$ & \multicolumn{1}{|c|}{ References } \\
\hline CON & 33 & $\begin{array}{l}9,11,14,16,26,28,66,109,114,115,116, \\
117,119,120,121,122,124,127,128,129, \\
130,132,134,135,138,142,145,157,185, \\
187,194,195,198\end{array}$ \\
\hline JOR & 23 & $\begin{array}{l}2,7,8,18,25,89,92,131,133,140,144, \\
146,150,151,154,156,177,179,180, \\
181,183,184,197\end{array}$ \\
\hline ART & 15 & $\begin{array}{l}81,118,123,125,126,136,143,155,176, \\
182,186,188,189,193,199\end{array}$ \\
\hline CP & 3 & $4,15,158$ \\
\hline RP & 3 & $95,190,196$ \\
\hline SUR & 2 & 191,192 \\
\hline BK & 1 & 10 \\
\hline WRK & 1 & 152 \\
\hline PUB & 1 & 6 \\
\hline CS & 1 & 13 \\
\hline PTH & 1 & 82 \\
\hline PRL & 1 & 175 \\
\hline & & \\
\hline Total & $\mathbf{8 5}$ & \\
\hline
\end{tabular}

\section{CONCLUSION AND LIMITATIONS}

The CC is a technology for providing different types of IT services which contain rental resources located in the cloud. The most common task present is increasingly conducted online for instance; checking of the emails, social media communication, editing and writing different types of documents, collaboration, watching videos and creating images. The study particularly focus on the trend of research on CC adoption internationally. Therefore, this study mainly aimed at identifying the different factors for the $\mathrm{CC}$ adoption by organizations in different sectors internationally. CC is increasingly being adopted, especially by many manufacturing, health, education, warehousing, engineering, telecommunication, construction and research sectors. In our analysis we have found that almost all the articles, even in different sectors of $\mathrm{CC}$ adoption, have at least addressed issues related to the adoption. Study findings particularly revealed that the utilisations of $\mathrm{CC}$ by companies is still an ongoing process in different sectors. Indeed, it was also clear that there is a different level of $\mathrm{CC}$ adoption by the companies in $\mathrm{SME}^{\mathrm{ec}} \mathrm{s}$ type's enterprises. Our findings have shown that internationally $\mathrm{CC}$ adoption is still an on-going 
process at different level in the companies, because some organisations have almost limited resources and financial capabilities to invest in IT. However, still there are significant security, factor influencing / affecting and challenging issues. These issues are analysed in most cases, which need to be tackledbefore $\mathrm{CC}$ services are effectively used by organizations internationally. Even though the literature review could not completely declare to be comprehensive, it still offers suitable awareness and allows the amount of research in the CC Adoption in SMEs.

Relevant on the effective use of $\mathrm{CC}$, that might be a quiet strategic element in the future competition of the organisations, is still insufficient. Whereas, standard are lacking, such as unified Application Programming Interface (APIs) and interoperability of various system, which is so far not granted. Therefore, the analysis of business impact looks at an early stage and for the assessment of benefits is not yet properly mature.

\section{A. Research Gaps}

This SLR has several limitations, because the review only analysed the 11 type of articles published in the period of 2009 to the first three quarters of 2018 in last ten years, those were generated based on a keyword search of "Cloud Computing Security" and "Cloud Computing Adoption". Hence it is only limited in keyword search methodology of the articles which mentioned the $\mathrm{CC}$ security and $\mathrm{CC}$ adoption. Whereas, the search criterion was limited to article title only; however, including abstracts as a criterion would have revealed more insightful articles. The literature only found from the 11 types of articles from 8types of databases from the internet. There must be other online databases containing more number of academic journal, articles, case studies, surveys, research papers, conceptual papers, press release, workshop, $\mathrm{PhD}$ theses, conference publications and book chapters in this particular subject, which might be able to give even a bigger exhaustive review of the 11 types of articles associated to the Adoption of CC in SMEs. On the other hand the only English publications were considered for the study of literatures. There must be publications in other foreign languages which may give a wider scope to further study.

\section{REFERENCES}

[1] Book: Cloud Computing: Web Based Dynamic IT Services. Author: Baun, Christian, Publisher: Springer - Date 14072011.

[2] Journal: Cloud Computing And Security Issues In Cloud. Authors LalitDashora, Aditya Jain, Gaurav Savner, AshutoshPatidar and Virendra Singh.

[3] Book: Cloud Security: A Comprehensive Guide to Secure Cloud Computing - Authors: Ronald L. Krutz and Russell Dean Vines.
[4] Conceptual Paper: The Cloud Adoption Toolkit: Supporting Cloud Adoption Decisions in the Enterprise. Authors Ali Khajeh-Hosseini, David Greenwood, James W. Smith, Ian Sommerville, $11^{\text {th }}$ August 2010.

[5] Book: Handbook of Cloud Computing: Authors BorkoFurht and Armando Escalante.

[6] Publication: Security and Privacy Issues in Cloud Computing. Author Jaydip Sen

[7] Journal: Cloud Computing And Security Issues In The Cloud. Authors Monjur Ahmed and Mohammad Ashraf Hossain.

[8] Journal: International Journal of Engineering Science and Technology (IJEST). Security Issues In Cloud Computing And Countermeasures. Authors Danish Jamil \& Hassan Zaki.

[9] Conference: On Technical Security Issues in Cloud Computing. Authors Meiko Jensen, JorgSchwenk, Nils Gruschka and Luigi Lo Iacono.

[10] Book: Cloud Security \& Privacy. Authors Tim Mather, Subra Kumaraswamy, \&Shahed Latif.

[11] Conference: Security Prospects through Cloud Computing by Adopting Multiple Clouds. Authors Meiko Jensen, JorgSchwenk, Jens-Matthias Bohli, Nils Gruschka and Luigi Lo Iacono.

[12] Article: Cloud Computing and Computing Evolution. Authors Markus Böhm, Stefanie Leimeister, Christoph Riedl, and Helmut Krcmar.

[13] Case Study: Factors That Affect The Adoption Of Cloud Computing For An Enterprise: A Case Study Of Cloud Adoption Within Intel Corporation. Authors Brian $\mathrm{T}$. McGeogh and Brian Donnellan.

[14] Conference: Adoption of Cloud Computing by SMEs in India: A study of the Institutional Factors. Completed Research Paper Author Jyoti M. Bhat.

[15] Conceptual Paper: Security Guidance for Critical Areas of Focus in Cloud Computing V2.1. Authors Jerry Archer, Alan Boehme, Dave Cullinane, Paul Kurtz, Nils Puhlmann and Jim Reavis. USA.

[16] International Conference: A Literature Review on Cloud Computing Adoption Issues in Enterprises. Author Rania El Gazzar.

[17] Book: Cloud Computing Implementation, management and Security, By John W. Rittinghouse and James F. Ranssome,

[18] Journal: An Overview of Cloud Computing System. Authors T. Krithiga and V. Poongodi.

[19] Article: Privacy Regulations for Cloud Computing Compliance and Implementation in Theory and Practice. Authors JoepRuiter and MartijnWarnier.

[20] Research Paper Public Cloud Computing vs. Private Cloud Computing: How Security Matters By Delvis Simmonds and AlliWahab.

[21] Article: Data Flow Management and Compliance in Cloud Computing. Authors Jatinder Singh, Julia Powles, Thomas Pasquier, and Jean Bacon.

[22] Journal: A Review On Issues And Challenges Of Cloud Computing. Authors Sangita Das, AnkitaChandrakar and Reshamlal Pradhan.

[23] Journal: Security and Authentication and Access on Data Transfer under the Cloud computing by using key. Author Saeed M. Hashim.

[24] Book: Conducting Research Literature Reviews: From the Internet to Paper (4th edition.). Author Arlene Fink, 2014.

[25] Journal: Cloud Computing The Business Perspective. Authors Sean Marston a, Zhi Li a ,SubhajyotiBandyopadhyay, Juheng Zhang and AnandGhalsasi.

[26] Conference: Cloud Computing: Transforming Medium and High Tech Industries in Kenya. Authors Pauline W. Wanjiku and Christopher A. Moturi.

[27] Journal: The Journal for Information Professionals. Author Nabil Sultan.

[28] Conference: Exploring Organizational Adoption of Cloud Computing in Singapore. Authors Margaret Tan \& Trisha TC Lin.

[29] Book: Research design: Qualitative, quantitative, and mixed methods approaches. By John W. 
[30] Journal: A Primer in Survey Research. Author Suzanne C. Watson.

[31] Journal article: Systematic planning for using an online survey, Authors Lois A. Ritter and Valerie M. Sue.

[32] Survey: The Cloud in 2017: Trends in Security, Author Riley Panko.

[33] Paper: Cloud Computing In Developing Economics. Author NirKshteri.

[34] Article: Roundup Of Cloud Computing Forecasts, 2017. Author Louis Columbus.

[35] Journal: What Is Coefficient Alpha? An Examination of Theory and Applications. Author Jose M. Cortina.

[36] Paper: Research Challenges for Enterprise Cloud Computing. Authors Ali Khajeh-Hosseini, Ian Sommerville and IlangoSriram.

[37] Report: CNBC: AWS revenue grows $42 \%$ to $\$ 4.10$ billion, by JorhanNovet.

[38] Press Release:Amazon.com Announces Second Quarter Sales up $25 \%$ to $\$ 38.0$ Billion, SEATTLE--(BUSINESS WIRE)-Jul. 27, 2017-- Amazon.com, Inc. (NASDAQ: AMZN) today announced financial results for its second quarter ended June 30,

2017.http://phx.corporateir.net/phoenix.zhtml?c=176060\&p=i rolnewsArticle \&ID=2289567

[39] Book: Data Analysis with SPSS: A First Course in Applied Statistic, Author Karen Grace-Martin and Stephen A. Sweet.

[40] Journal: Effects of Industry Type on ICT Adoption among Malaysian SMEs. Authors Khong Sin Tan ,Uchenna Cyril Eze, and Siong Choy Chong.

[41] Paper: How large are software cost overruns? A review of the 1994 CHAOS report. Authors MagneJørgensen and KjetilMoløkken.

[42] Book: Information Security Fundamentals. Author Thomas R. Peltier.

[43] Conference: A case of Pakistan," Cloud Computing and Intelligence Systems (CCIS), (pp. 536-540). Authors Khan, S., Zhang, B., Khan, F., \& Chen, S.

[44] Paper: Cutting Through The Fog: Understanding The Competitive Dynamics In Cloud Computing, BRIE Working Paper 190 (Beta) May 1, 2010 cCopyright 2010. Authors Kenji E. Kushida, Dan Breznitz and John Zysman.

[45] Journal: Contemporary trends and issues in IT adoption and diffusion research. Authors Michael D Williams, Yogesh K Dwivedi, Banita Lal, Andrew Schwarz.

[46] Article: "Blanket" approaches to promoting ICT in small firms: some lessons from the DTI ladder adoption model in the UK". Authors Article citation: Lynn M. Martin, Harry Matlay.

[47] Book: Research methodology: Methods and techniques. Author Kothari.

[48] Book: Research methods for business: A skill building approach, Author Sekaran, U.

[49] PhD Theses: Implementing a Forensic Educational Package for Registered Nurses in Two Emergency Departments in Western Australia (Doctor of Philosophy (PhD). By Michel, C. M.

[50] Book: Research methods for business students (5th edition). Author Saunders, M. N., Lewis, P., \& Thornhill, A.

[51] Book: Foundations of qualitative research: Interpretive and critical approaches. Author Willis, J. W.

[52] Book: Social Research Methods: Qualitative and Quantitative Approaches. (C) Pearson Education Limited 2014 (7th edition). USA.

[53] Book: Competing paradigms in qualitative research. In N. K. Denzin \& Y. S.

[54] Book: Paradigmatic controversies, contradictions, and emerging confluences. Authors Lincoln, Y. S., Lynham, S. A., \& Guba, E. G. (2011). USA: Sage Publications.

[55] Paper: Exploring the philosophical underpinnings of research: relating ontology and epistemology to the methodology and methods of the scientific, interpretive, and critical research paradigms. Author Scotland, J.

[56] Book: Sociology (14th edition). Author John J. Macionis.

[57] Book: Research design: Qualitative, quantitative, and mixed methods approaches (4th edition). Author Creswell, J. W.
[58] Book: Ethnographic research: A guide to general conduct. Author Ellen, R. F.

[59] Book: Critical approaches to the study of higher education: A practical introduction. Authors Martínez- Alemán, A. M., Pusser, B., and Bensimon, E. M.

[60] Book: Educational Research: Quantitative, Qualitative, and Mixed Approaches, Authors Johnson, B., \& Christensen, L.

[61] International Journal: Good Practice In The Conduct And Reporting Of Survey Research. Authors Kelly, K., Clark, B., Brown, V., \&Sitzia, J.

[62] Book: Introduction to research methods in education. Author Punch, K. F.

[63] Book: Management Research Methods. Authors Tharenou, P., Donohue, R. and Cooper, B.

[64] Book: Multivariate data analysis. (Seventh Edition), Authors Joseph F. Hair Jr., William C. Black, Barry J. Babin and Rolph E. Anderson.

[65] SPSS Survival Manual: A Step by Step Guide to Data Analysis Using SPSS. By Pallant, J., Allen \& Unwin.

[66] Conference: Impacts On The Organizational Adoption Of Cloud Computing: A Reconceptualization Of Influencing Factors. Authors Mark Stieningera, DietmarNedbala, Werner Wetzlingera, GeroldWagnera and Michael A. Erskineb.

[67] Journal: Determinants of Small Business EDI Adoption: An Empirical Investigation. Author Chau, P. Y., \& Hui, K. L.

[68] Research Article: Virtualization In Cloud Computing. Author T. Swathi, K. Srikanth and S. Raghunath Reddy.

[69] Journal: Cloud Computing: Centralization and Data Sovereignty. Authors Primavera De Filippi and Smari McCarthy.

[70] Journal: High-Level Managerse Considerations for RFID Adoption in Hospitals: An Empirical Study in Taiwan. Authors, Hui-Min Lai \& I-Chun Lin \& Ling-Tzu Tseng.

[71] Journal: Review on IT Adoption: Insights From Recent Technologies. Authors HemlataGangwar, Hema Date and A.D. Raoot.

[72] Book: Integrated Series in Information Systems Volume 28, Information Systems Theory, Explaining and Predicting Our Digital Society, Vol. 1, Authors Yogesh K. Dwivedi, L Michael R. Wade and Scott L. Schneberger.

[73] Book: Diffusion of Innovations, Author Rogers, E. M. New York: Free Press.

[74] Presentation: Research Methods, Author William G. Zikmund.

[75] Article: SMEs In The Cloud: The Impact Of Cloud Adoption On Economic Growth And Development. Authors EvangeliaFiliopoulou, PersefoniMitropoulou and Christos Michalakelis.

[76] Conference: Costing of Cloud Computing Services: A Total Cost of Ownership Approach. Authors Benedikt Martens, Marc Walterbusch and Frank Teuteberg.

[77] Dr.Ramnath K. Chellappa, Associate Professor \& Caldwell Research Fellow Doctoral Program Coordinator, Information Systems \& Operations Management, Room 407, 1300 Clifton Road, Goizueta Business School, Emory University, Atlantaa. GA 30322-2710. (DEFINITION IN SECTION: CLOUDCOMPUTING)http://www.bus.emory.edu/ram/

[78] Conference: Managing Cloud Computing: A Life Cycle Approach. Authors Gerard Conway and Edward Curry.

[79] History of Cloud Computing Timeline. https://cdn2.hubspot.net/hubfs/620276/History_of_Cloud_Co mputing_Timeline.pdf? $\mathrm{t}=1488486871142$ [80]Paper: The Economic Impact of Cloud Computing on Business Creation, Employment and Output in Europe. Author Federico Etro.

[81] Article: A Brief History of Cloud Computing. Author Keith D, Foote.

[82] Dot-com bubble, from Wikipedia. https://en.wikipedia.org/wiki/Dot-com_bubble

[83] Article: Service Level Management (SLM) in Cloud Computing Third party SLM framework. Authors GianmarioMotta, Linlin You, Nicola Sfondrini, Daniele Sacco and Tianyi Ma. 
[84] Article: Adoption of Software as a Service (SaaS) Enterprise Resource Planning (ERP) Systems in Small and Medium Sized Enterprises (SMEs). Author Ravi Seethamraju.

[85] Conference: Costing of Cloud Computing Services: A Total Cost of Ownership Approach. Authors Benedikt Martens, Marc Walterbusch and Frank Teuteberg.

[86] Conference: A comparative study of major service providers for cloud computing. Authors Noman Islam and AqeelurRehman.

[87] Book: What is Cloud Computing, Basic of Cloud Computing (PDF). Author Sourabh,

[88] Paper: Centre For Economics And Business Research Ltd THE CLOUD DIVIDEND: Part One. The Economic Benefits of Cloud Computing to Business and the Wider EMEA economy-France, Germany, Italy, Spain and the UK (December 2010), 89.

[89] Journal: Secure Database as a Service-a Review. Authors Prof SwarnalataBollavarapu and Kamal Mistry.

[90] National Institute of Standards and Technology (NIST),Definition of Cloud Computing. Recommendations of the National Institute of Standards and Technology. Author Peter Mell and Timothy Grance,

[91] Paper: Addressing cloud computing security issues. Authors Dimitrios Zissis and DimitriosLekkas.

[92] Journal: Public vs Private vs Hybrid vs Community - Cloud Computing: A Critical Review. Author Sumit Goyal. I.J.

[93] IEEE International Conference: Cloud Computing: Issues and Challenges. Authors Tharam Dillon, Chen Wu and Elizabeth Chang.

[94] Book: Architectecting the Cloud: Design Decisions for Cloud Computing Service Models (SaaS, PaaS and IaaS). Author Kavis, M (2014). John Wiley.

[95] Research Paper: Adoption Of Cloud Computing and Services - An Objective Analysis. Author Srinivas R Kondapalli.

[96] Research Journal: Analysis of Trend, Service and Deployment Models in Cloud Computing with Focus on Hybrid Cloud and its Implementation. Authors Praveena, D. and Rangarajan, P.

[97] Article: Research Article A Comprehensive Study on Cloud Computing. Authors Suruchee V. Nandgaonkar ,Prof. A. B. Raut.

[98] Book: Intelligent Data analysis and its Applications, Volume I, (C) 2014. Editors: Pan, J.-S., Snasel, V., Corchado, E.S., Abraham, A., Wang, S.-L.

[99] Article: From outsourcing to Cloud computing: evolution of IT services. Author SubhankarDhar.

[100] Journal: Cloud computing: state-of-the-art and research challenges. Authors Zhang, Q., Cheng, L. \&Boutaba, R.

[101] Paper: A "cloud lifestyle": The diffusion of cloud computing applications and the effect of demographic and lifestyle clusters. Authors Coursaris, C. K., van Osch, W., \& Sung, J.

[102] Book: Limitations and challenges in cloud-based applications development. Authors Pramod, N., Muppalla, A. K., \&Srinivasa, $\mathrm{K}$.

[103] Conference: International Conference Paper Cloud computing security issues and challenges: Authors Verma, A., \& Kaushal, S.

[104] The Technology-Organization-Environment Framework. Author Baker, J. (2012). In: Y. K. Dwivedi, M. R. Wade, \& S. L. Schneberger.

[105] Journal: Security on distributed systems: Cloud security versus traditional IT. Authors Himmel, M.A. \& Grossman, F.

[106] Journal: A survey study on major technical barriers affecting the decision to adopt cloud services. Authors Phaphoom, N., Wang, X., Samuel, S., Helmer, S. \&Abrahamsson, P.

[107] Article: Integrating quantitative and qualitative research: How is it done? Author Bryman, A.

[108] Book: Designing and conducting mixed methods research. Authors John W. Creswell, Vicki L. Plano Clark. Sage Publications. $19^{\text {th }}$ Sep 2017.

[109] Conference: Impact Study Of Cloud Computing On Business Development. Author C. Lakshmi Devasena.

[110] Article: Seven Indisputable Technology Trends That Will Define 2015 Stephen J. Andriole

[111] White Paper: Understanding and Supporting Cloud Computing Adoption in Irish Small- and Medium-Sized
Enterprises. By Dr Marian Carcary, Dr Eileen Doherty, Gerard Conway.

[112] Conference: Digital Signature as a Cloud-based Service. Author WojciechKinastowski.

[113] Technical Report: Guidelines for performing Systematic Literature Reviews in Software Engineering. Authors Barbara Kitchenham, Pearl Brereton, David Budgen, Mark Turner, John Bailey and Stephen Linkman.

[114] Conference: Heading for the Clouds? Implications for Cloud Computing Adopters. Authors Abokhodair, N., Taylor, H., Hasegawa, J., Mowery, S.J.:

[115] Conference: Advantages and challenges of adopting cloud computing from an enterprise perspective. Author Maricela Georgiana Avram (Olaru).

[116] Conference: Exploring the Impact of Cloud Computing Adoption on Organizational Flexibility: A Client Perspective. Authors Sangeeta Shah Bharadwaj and Prerna Lal.

[117] Conference: Cloudrise: Exploring Cloud Computing Adoption and Governance With the TOE Framework. Authors Hans P. Borgman, BouchaibBahli, HaukeHeier and Fiona Schewski.

[118] Articles: Cloud computing adoption in Greece. Authors YaznAlshamaila, SavvasPapagiannidis and TetaStamati.

[119] Conference: Factors Influencing an Organisation's Intention to Adopt Cloud Computing in Saudi Arabia. Author NoufAlkhater, Gary Wills and Robert Walters.

[120] Conference: Cloud Computing Adoption Assessment Model (CAAM). Author Usman Nasir, \& Mahmood Niazi.

[121] Conference: Four Scenarios for Adoption of Cloud Computing in China. Authors Luoma, E. and Nyberg, T.:

[122] Conference: Cloud Computing Adoption by Firms. Authors Espadanal, M. and Oliveira, T.

[123] Article: Prospect Of Adopting Cloud Computing In Indian Manufacturing Industry. Authors Prof.Supriya Santosh. Wagh and Research Scholar, Dr. D.Y. Patil.

[124] Conference: Decision Points for Adoption Cloud Computing in Small, Medium Enterprises (SMEs). Authors Ashwini Rath, Sanjay Mohapatra, Sanjay Kumar and Rahul Thakurta.

[125] Article: Identification Of A Company 's Suitability For the Adoption of Cloud Computing and Modelling Its Corresponding Return on Investment. Authors Subhas Chandra Misra and ArkaMondal.

[126] Article: Towards an Understanding of Cloud Computingees Impact on Organizational IT Strategy. Authors Ruoning Qian and Prashant Palvia.

[127] Conference: Factors Affecting The Adoption Of Cloud Computing: An Exploratory Study. Authors Morgan Lorraine, and Conboy, Kieran.

[128] European Conference: Analysis of Dissatisfiers That Inhibit Cloud Computing Adoption Across Multiple Customer Segments. Authors Easwar Krishna Iyer, Arathi Krishnan, Gaurav Sareen and Tapan Panda.

[129] International Conference: An Exploratory Analysis of the Influence of Information Security on the Adoption of Cloud Computing. Authors Omondi John Opala, Syed M. Rahman.

[130] International Conference: Cloud Computing Adoption And Its Implications For Cio Strategic Focus - An Empirical Analysis. Authors Suresh Malladi, and M.S. Krishnan.

[131] Journal: Cloud Computing as an Innovation: Percepetion, Attitude, and Adoption. Authors Angela Lin and Nan-Chou Chen.

[132] European Conference: Contributing Factors of Cloud Computing Adoption: a Technology-Organisation Environment Frameork Approach. Authors Matews Z. NkhomaandDuy P.T. Dang.

[133] International Journal: The Usage And Adoption Of Cloud Computing By Small And Medium Businesses. Authors Prashant Gupta, A. Seetharamana and John Rudolph Raj.

[134] Conference: Will Adoption of Cloud Computing Put the Enterprise at Risk? Authors UgochukwuOnwudebeluand Benedict Chukuka.

[135] Conference: Adoption of Cloud Computing in Organizations. Author Hasan Nuseibeh.

[136] Article: Adoption of Cloud Computing Technologies in Supply Chains: An Organizational Information Processing 
Theory. Authors Cegielski, C.G., Jones-Farmer, L.A., Wu, Y. and Hazen. B.T.

[137] Article: User Centric Cloud Service Model in Public Sectors: Author Shin, Sungkyunkwan

[138] Conference: The Viability of Cloud Computing Adoption in SMME"s in Namibia. Authors Ricardo Tjikongo, and Walter Uys.

[139] Article: Cloud Computing. Author By Brian Hayes.

[140] Journal: An Exploratory Study To Understand The Critical Factors Affecting The Decision To Adopt Cloud Computing In Taiwan Hospital. Authors JiunnWoeiLian,David C. Yenand Yen-Ting Wang.

[141] Journal: Factors affecting the adoption of electronic signature: Executives' perspective of hospital information department. Authors Chang, I. C., Hwang, H. G., Hung, M. C., Lin, M. H., and Yen, D. C.

[142] Conference: The determinants of Cloud Computing Adoption by Large European Firms. Authors Xiaolin Cheng and Ahmed Bounfour.

[143] Article: The Slow Adoption of Cloud Computing and IT Workforce. Authors Muhammad H. Raza, Adewale Femi Adenola, Ali Nafarieh and William Robertson.

[144] Conference: Adoption Issues for Cloud Computing. Authors Won Kim, Soo Dong Kim, Eunseok Lee and Sungyoung Lee.

[145] Conference: Understanding the Determinants of Cloud Computing Adoption for High Performance Computing. Authors Theo Lynn, Xiaoning Liang, Anna Gourinovitch, John P. Morrison, Grace Fox and PierangeloRosati.

[146] Journal:. Factors Affecting Cloud Computing Adoption in a Developing Country - Ghana: Using Extended Unified Theory of Acceptance and Use Of Technology (UTAUT2) Model Authors Rebecca AdwoaAmponsah, Joseph KobinaPanford and James Ben Hayfron-Acquah.

[147] Conceptual Paper: Interoperability and Portability for Cloud Computing: A Guide Version 2.0. Authors Claude Baudoin, Eliezer Dekel, Mike Edwards, John Meegan Jem Pagan, Karolyn Schalk, John Shortt, Gurpreet Singh, Joe Talik, William Van Order Annie Sokoland Steven Woodward.

[148] Book: Basic Cloud Computing Types. In: Cloud Computing Basics. Author Srinivasan, S. First online $15^{\text {th }}$ May 2014.

[149] Conference: Secure Cloud Computing Benefits, Risks and Controls. Authors Mariana Carroll, Alta van der Merwe and Paula Kotzé.

[150] Journal: Assessing The Determinants Of Cloud Computing Adoption: An Analysis Of The Manufacturing And Services Sectors. Authors Tiago Oliveira, Manoj Thomas and Mariana Espadanal.

[151] Journal: Benefits And Challenges Of The Adoption Of Cloud Computing In Business. Authors Colin Ting Si Xue and Felicia Tiong Wee Xin.

[152] Workshop: Identifying Cloud Security Threats to Strengthen Cloud Computing Adoption Framework. Authors NabeelKhana and Adil Al-Yasirib.

[153] Conference: Research Gaps And Trends In Cloud Computing: A Systematic Mapping Study. Authors Liping Zhao, Liang-Jie Zhang and Tina X. O. Liu.

[154] Journal: Factors Influencing Cloud Computing Adoption In Small And Medium Enterprises. Authors Haslinda Hassan, MohdHerryMohd Nasir, NorhaizaKhairudin\& Iskandar Adon.

[155] Article: Factors Influencing Cloud Computing Adoption in the Public Sector: An Empirical Analysis. Authors HasimiSallehudin, RazliCheRazak and Mohammad Ismail.

[156] Journal: The Influence Of Information Security On The Adoption Of Cloud Computing: An Exploratory Analysis. Authors Omondi John Opala, Shawon Rahman and AbdulhameedAlelaiwi.

[157] Conference: Cloud computing adoption determinants: an analysis of Australian SMEs. Authors Salim Al Isma'ili, Mengxiang Li, Jun Shen and Qiang He.

Dicken, Tay Xin Hui, TengMee Ling and MohdSanusiAzmi. DOI: $10.30630 /$ joiv.2.4-2.171.
[158] Conceptual Paper:Adoption And Implementation Of Cloud Computing Services: A Railroad Company Case. Authors Dalsang Chung and Sun Gi Chun.

[159] Journal: A Comparison between Centralized and Distributed Cloud Storage Data- Center Topologies. Author Maurice Bolhuis.

[160] Conference: Predicting SMEs willingness to adopt ERP, CRM, SCM \& E-procurement systems. Authors Ben Ramdani and Peter Kawalek.

[161] Journal: Risks in Enterprise Cloud Computing: the Perspective of IT Experts. Authors Arnab Dutta, Guo Chao Alex Peng and AlokChoudhary.

[162] Article: Diffusing the Cloud: Cloud Computing and Implications for Public Policy. Authors Kenji E. Kushida\& Jonathan Murray \& John Zysman.

[163] Article: AnalyzingThe Past To Prepare For The Future: Writing A Literature Review. Authors Jane Webster and Richard T. Watson.

[164] Journal: A Systems Approach to Conduct an Effective Literature Review in Support of Information Systems Research, Authors Yair Levy and Timothy J. Ellis.

[165] International Conference: A Concept Map of Information Systems Research Approaches. Authors Rafael Gonzalez, and AjanthaDahanayake.

[166] Journal Online: The Online Journal of McKinsey \& Co. Delivering software as a service. Authors Abhijit Dubey and DilipWagle.

[167] Article: A Descriptive Literature Review and Classification of Cloud Computing Research. Authors Haibo Yang and Mary Tate.

[168] Working Paper: A Guide to Conducting a SLR of Information Systems Research. Authors ChituOkoli and Kira Schabram.

[169] Conference: Reconstructing The Giant: On The Importance Of Rigour In Documenting The Literature Search Process. Authors Jan VomBrocke, Alexander Simons, BjoernNiehaves, Bjorn Niehaves, Kai Reimer, Ralf Plattfaut\& Anne Cleven.

[170] Article: Studying Information Technology in Organizations: Research Approaches and Assumptions. Authors Wanda J. Orlikowski and Jack J. Baroudi.

[171] Paper: Choosing Systems Appropriate Research Information Methodologies. Authors Robert D. Galliers and Frank F. Land.

[172] Research Article: Using grounded theory as a method for rigorously reviewing literature. Authors Joost F. Wolfswinkel, ElfiFurtmueller and Celeste PM Wilderom.

[173] Conference: An Understanding of Business Process Standardization. Authors KanikaGoel, Dr.WasanaBandara and Prof. Guy GableIn.

[174] Paper: Cloud Computing Strategy Direction Paper. By the Department of Finance and Deregulation (DFD) Cloud Computing Strategic, by the Australian Government.

[175] Press Release: Gartner Forecasts Worldwide Public Cloud Revenue to Grow 17.3 Percent in 2019. Stamford, Conn., September 12, 2018. USA. To contact Katie Costello, Gartner, Sarah Hippold, Gartner.https://www.gartner.com/en/newsroom/pressreleases/201809-12-gartner-forecasts-worldwide-publiccloud-revenue-togrow-17-percent-in-2019

[176] Article: Factors That Influence Adoption of Cloud Computing: An Empirical Study of Australian SMEs. Authors Ishan Senarathna, Carla Wilkin, Matthew Warren, William Yeoh and Scott Salzman.

[177] International Journal: Challenges of Cloud Computing Adoption From the TOE Framework Perspective. Authors, Omar Al-Hujran, Enas M. Al-Lozi, Mutaz M. Al Debei, Mahmoud Maqableh.

[178] Book: Diffusion Of Innovations. Author Everett M. Rogers. Third Edition, 1983.

[179] Journal: A Review on Cloud Computing Security. Authors Marry Teo, HairulnizamMahdin, Lee JiaHwee, Haezel Ann

[180] Journal: Adoption of Cloud Computing among Enterprises in Malaysia. Authors Kamal Karkonasasi, Ahmad 
SuhaimiBaharudin, BabakEsparham and SeyedAliakbar Mousavi.

[181] Journal: Factors Influencing The Organizational Adoption Of Cloud Computing: A Survey Among Cloud Workers. Authors Mark Stieninger, Gerold Wagner, DietmarNedbal, Michael A. Erskine and Werner Wetzlinge.

[182] Article: Challenge of Cloud Computing for SMEs: A Case of Baltic Countries. Authors KarlisKreslins, Darya Novik and TatjanaVasiljeva.

[183] Journal: Factors That Affect Cloud Computing Adoption by Small and Medium Enterprises In Kenya. Author John Ngugi Makena.

[184] Journal: Factors Influencing Cloud Computing Adoption in Saudi Arabiaes Private and Public Organizations: A Qualitative Evaluation. Author Mohammed AteeqAlanezi.

[185] Conference: Organisational factors affecting cloud computing adoption in small and medium enterprises (SMEs) in service sector. Author Haslinda Hassan.

[186] Article: Key Management Determinants for Cloud Computing Adoption. Authors Dr Anabel Gutierrez and J. Ranald. I. Lumsden.

[187] Conference: Key Issues for the Successful Adoption of Cloud Computing. Authors TeófiloBrancoJr.a, Filipe de Sá-Soaresa and Alfonso Lopez Rivero.

[188] Article: Cloud Computing Adoption in Organisations: Review of Empirical Literature. Authors Haslinda Hassan, MohdHerryMohd Nasir and NorhaizaKhairudin.

[189] Article: Determinants of cloud computing adoption at firm level: From the technological context. Authors Haslinda Hassan and MohdHerryMohd Nasir.

[190] Research Paper: The Importance of Business Model Factors for Cloud Computing Adoption: Role of Previous
Experiences. Authors Kristina BogatajHabjan and Andreja Pucihar.

[191] Survey: Right Scale 2018 State of the Cloud Report ${ }^{\mathrm{TM}}$. DATA TO NAVIGATE YOUR MULTI-CLOUD STRATEGY.

USA, 2018.

[192] Survey: Public Cloud Adoption in Multinational Companies A survey. Author Nicola Sfondrini. Italy, 2018.

[193] Article: Sustainable Cloud Computing: Foundations and Future Directions. Authors RajkumarBuyya and Sukhpal Singh Gill.

[194] Conference: A Review Study on Cloud Computing Issues. Authors Qusay Kanaan Kadhim, RobiahYusof, Hamid Sadeq Mahdi, Sayed Samer Ali Al-shami and SitiRahayuSelamat.

[195] Conference: Key Issues for Embracing the Cloud Computing to Adopt a Digital Transformation: A study of Saudi Public Sector. Authors Majid Al-Ruithe, ElhadjBenkhelifa and Khawar Hameed.

[196] Research Paper: A Survey of Cloud Computing Security: Issues, Challenges and Solutions. Authors ElomWorlanyo and Prof. Raj Jain.

[197] Journal: Network Security: Security in Cloud Computing. Authors Mr. Vikas Malik, Srishti Gupta and Jyoti Kaushik.

[198] +Conference: A Comprehensive Study On Cloud Computing. Authors Ab Rashid Dar and Dr. D. Ravindran.

[199] Article: Reviews on Security Issues and Challenges in Cloud Computing. Authors Y Z An, Z F Zaaba\& N F Samsudin.

[200] Report:9 Cloud Computing Security Risks Every Company Faces, And What Proactive Steps You Can Take to Protect Your Data.AuthorCameronColes.

https://www.skyhighnetworks.com/cloud-security-

blog/9cloud-computing-security-risks-every-company-faces/ 